\title{
Studies of Static and Dynamic Characteristics of the EDM Machine Based on the ANSYS Workbench
}

\author{
J. H. Wang, ${ }^{a}$ G. Li, ${ }^{a, 1}$ Z. F. Liu, ${ }^{a}$ Y. S. Zhao, ${ }^{a}$ Z. J. Cheng, ${ }^{a}$ J. Y. Liu, ${ }^{b}$ and Y. Li ${ }^{\text {b }}$ \\ ${ }^{a}$ College of Mechanical Engineering and Applied Electronics Technology, Beijing University of \\ Technology, Beijing, China \\ ${ }^{\mathrm{b}}$ Beijing Institute of Electro-Machining, Beijing, China \\ 1523455334@qq.com
}

The static and dynamic characteristics of the EDM machine as the main machining unit directly influence the machining accuracy and stability. For a particular type of the EDM machine, the three-dimensional model was built with SolidWorks and the finite element method was employed with the ANSYS Workbench to analyze the static and dynamic characteristics of the machine. The results show that the stiffness of the ram and vertical guide (Z-axis) in the $Y$ and $Z$ directions is the weak side of the machine. The analytical method is proved to be effective for the optimization of the machine design.

Keywords: EDM machine, geometry model, finite element model, static and dynamic analyses.

Introduction. With the rapid development of the aviation and aerospace industry, EDM machine tools have been used widely in the field of aerospace, for their high precision, excellent processing properties of machining complex cavities and narrow spatial structures [1]. In the design of machine tools, the static and dynamic properties are the key evaluation criteria, which need to be taken into consideration during the design process. For the static and dynamic characteristics, the finite element method (FEM) was widely applied by researchers of the country and abroad. Myers [2] used FEM to study the structural dynamics of the vertical grinder and obtained its static and dynamic stiffness characteristics. Zhang et al. [3] constructed the finite element model of CX8075 vertical machining center based on ANSYS workbench software to analyze the dynamic performance of key parts. Kang and Fan [4] performed the model analysis and harmonic response analysis of an ZK7640 column by using ANSYS software and laid the foundation for an optimized design and remanufacturing. Feng et al. [5] built five worktable models with different stiffening ribs using SolidWorks software and calculated harmonic response of each model by ANSYS workbench software to get the best solution. Li et al. [6] made the model analysis of the EDM machine on the platform of ANSYS workbench software. Li [7] performed the transient dynamic analysis of the SEDM model with ANSYS and then proposed the modification program. Shi et al. [8] made the static and modal analysis of the HTM40100 horizontal turning-milling machine center and discovered its weak side.

Most of these researchers used finite element software to implement the modular analysis of machine tools, but could hardly consider the characteristics of each module in verifying the correctness of the entire analysis. This article presents the study on a particular type of the EDM machine as an example of describing the process of analysis.

In the first section, it discusses the method of establishing the finite element model. At first, the three-dimensional geometry model of the machine is established with SolidWorks software. Then the simplified model is meshed by importing into ANSYS workbench software. After that, the second section covers the efficiency of the machine structure. The static analysis is conducted to obtain the deformation and equivalent stress nephograms. In the last section, the dynamic analysis, which includes the modal and harmonic response 
ones, is carried out to reveal the weak side of the machine. Here the mode shapes and the frequency response deformation curve of the whole machine are obtained, which contributes to understanding the machine vibration performance and confirms the optimization direction of the machine structure.

\section{Finite Element Model Construction.}

1.1. Three-Dimensional Geometry Model of the Machine. The selected EDM machine belongs to Ngau-Tau-type. It is a kind of a die-sinking EDM machine, which is made up of several parts: the column is bolted on the bed, which is attached to the ground by anchor bolts. The sliding plate and the ram are connected by the rail and slider to realize the movement in the $X$ and $Y$ directions. The $Z$-axis base is installed on the top of the ram using the fastening bolts. The $Z$-axis is connected to its base by the rail and slider to realize the movement in the $Z$ direction, where the electrode mounting plate is fastened. This structure has the advantages of strong bearing capacity and good installation stability of a workpiece. Its machining precision would not be affected by the worktable under loading. In the process of design, analysis of the key parts cannot represent the overall performance of the machine. Therefore, the finite element analysis must be carried out to get accurate predictions of the EDM machine.

The construction of the three-dimensional geometry model of the machine tool is the premise of the finite element analysis. The machine tool has too many complex elements to construct the finite element models accurately enough. Therefore, it is necessary to simplify the structure for the construction of the three-dimensional model. Specifically, the simplified principles should cover the two following aspects:

(i) neglecting the chamfers, fillets, small bolt holes and other small pieces;

(ii) making the small tapers, narrow flat surfaces straight and planar.

After simplification, the physical properties of the whole structure would not be changed, but it provides a premise for subsequent analysis and calculation efficiency. SolidWorks software can realize the seamless connection with ANSYS workbench, and the model built with it can be directly imported into ANSYS software, so that the process can greatly reduce the model transformation error. Firstly, the simplified model of the key components is built with SolidWorks, and then assembled into a whole to import into ANSYS workbench. The imported model is shown in Fig. 1. The position shown in Fig. 1 is the extreme machining position, that is to say, the tool electrode is located at the maximum vibration position: the sliding plate moves to the forefront of the travel, and the Z-axis moves to the bottom of the stroke.

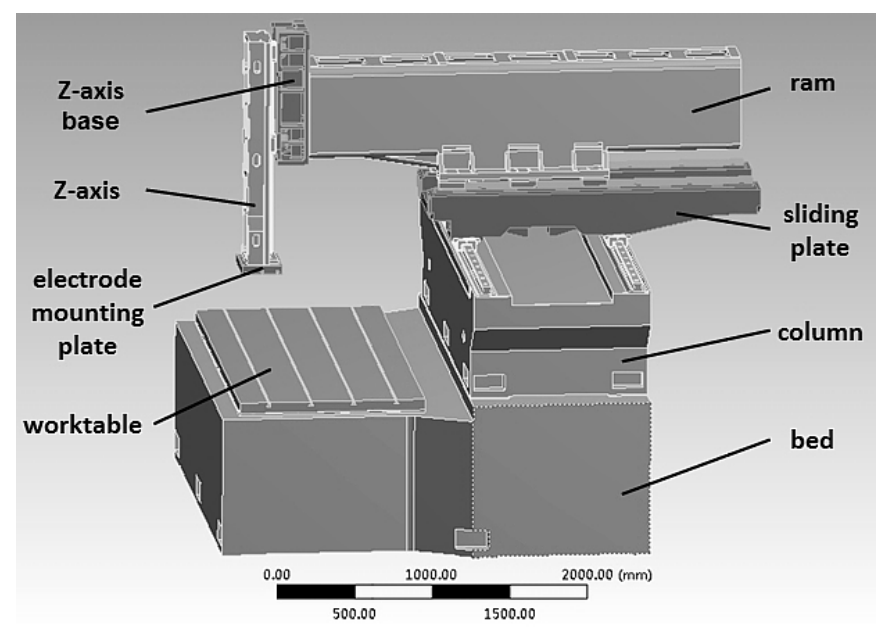

Fig. 1. Three-dimensional geometry model of the EDM machine. 
1.2. Definition of Material Properties and Meshing. After importing the geometry model, the next step is to set the material properties of the key parts. The bed, column, sliding plate, ram, base of $Z$-axis, and $Z$-axis are all made of gray cast iron, and the material of the electrode mounting plate is structural steel. The rail and slider are chosen THK products, which are made of stainless steel. To guarantee good insulation and minimum deformation, natural granite is selected as the material of the worktable. The specific parameters are listed in Table 1.

$\mathrm{T}$ a

Material Properties of Key Parts

\begin{tabular}{|c|c|c|c|}
\hline Key part & $\begin{array}{c}\text { Elastic } \\
\text { modulus (Pa) }\end{array}$ & $\begin{array}{c}\text { Poisson's } \\
\text { ratio }\end{array}$ & $\begin{array}{c}\text { Density } \\
\left(\mathrm{kg} / \mathrm{m}^{3}\right)\end{array}$ \\
\hline $\begin{array}{c}\text { Bed, column, sliding plate, ram, } \\
\text { Z-axis base, and Z-axis }\end{array}$ & $1.38 \cdot 10^{11}$ & 0.3 & 7200 \\
\hline $\begin{array}{c}\text { Electrode mounting plate, rail, } \\
\text { and slider }\end{array}$ & $2.07 \cdot 10^{11}$ & 0.3 & 7850 \\
\hline Worktable & $0.5 \cdot 10^{11}$ & 0.3 & 2800 \\
\hline
\end{tabular}

For the combined faces, the module of "automatic generation of the contact surface" is chosen, which can quickly identify the contact relationship between the two parts. For example, the screw fixation connected faces belong to the rigid connection, so the type of connection is called "bonded," while the rail and slider connected faces are a kind of the movement contact surface, whose type is "frictionless."

Meshing is an important part of the finite element model construction. The number of nodes directly affects the accuracy and scale number of calculations. The "automatic meshing function" is adopted. For calculations, the tetrahedral structure is chosen to simulate the complex and irregular one. After comparison, the Solid187 unit is selected, which is a high-order 3D structure with 10 nodes. It exhibits the quadratic displacement mode and can better simulate the irregular models. The element size is set to 20, and hex dominant is selected as the division method. The finite element model after meshing is shown in Fig. 2. There are 1254,961 nodes and 381,843 units. Thus, the finite element model has been created.

2. Static Analysis of the Whole Machine. For static analysis, the preprocessing is required, including supports and loads. Firstly, the bed is fastened to the ground with anchor bolts, which should be fixed firmly to ensure the machining stability. For these reasons, the type of a support is defined as the "fixed support." Secondly, the main spindle component precision of a Ngau-Tau-type EDM machine is greatly affected by gravity, so it is necessary to consider the whole weight of the machine structure. The liquid weight $G$ should also be accounted due to the whole infusion processing pattern of the EDM machine. Finally, the electrode is installed at the top of the electrode mounting plate, whose maximum weight is $300 \mathrm{~kg}$. All the constraint conditions are shown in Fig. 3. Among them, the computational formula $G$ is

$$
G=\gamma \rho g V=1.2 \cdot 0.8 \cdot 10^{3} \cdot 9.8 \cdot 4.02 \approx 37820 \mathrm{~N}, \quad \text { approximately round } 40 \mathrm{kN},
$$

where $\gamma$ is the safety factor taken to be 1.2, $\rho$ is the density of the EDM machining liquid equal to $0.8 \cdot 10^{3} \mathrm{~kg} / \mathrm{m}^{3}, g$ is the gravity equal to $9.8 \mathrm{~m} / \mathrm{s}^{2}, V$ is the maximum machining liquid volume, $V \approx 4.02 \mathrm{~m}^{3}$. 


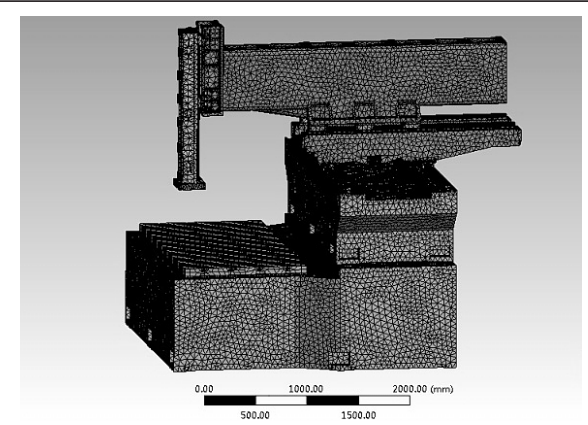

Fig. 2. Finite element model of the EDM machine.

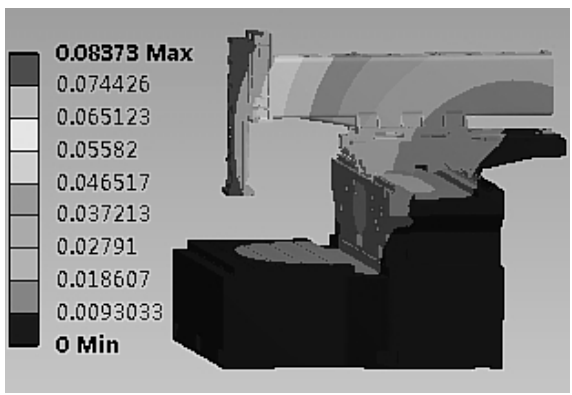

a

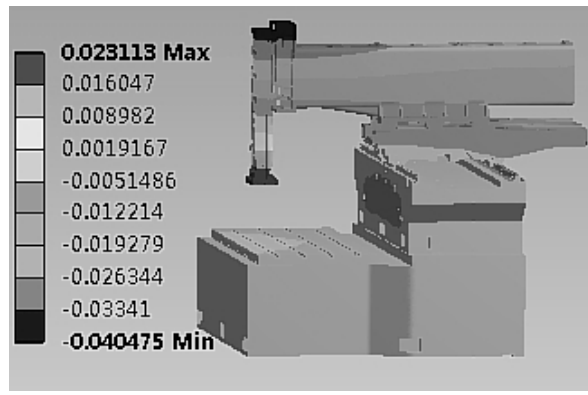

$\mathrm{c}$

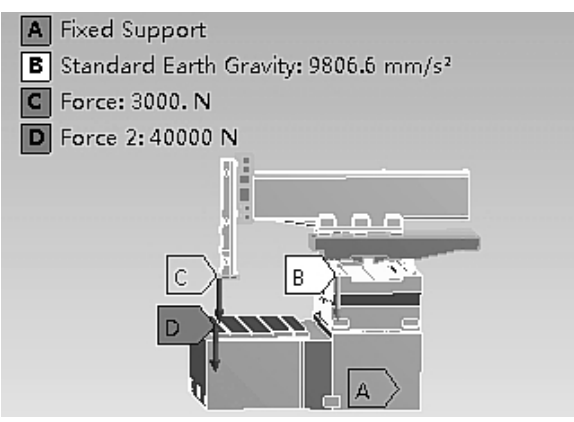

Fig. 3. Supports and loads.

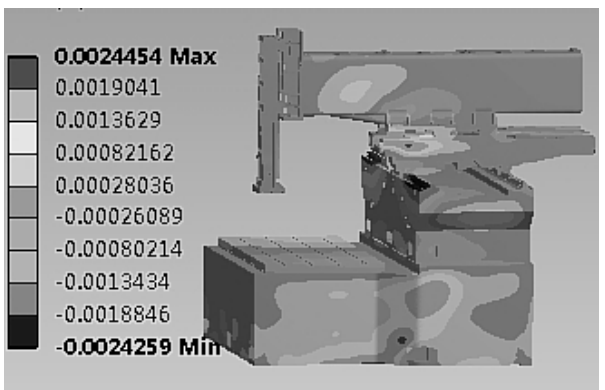

b

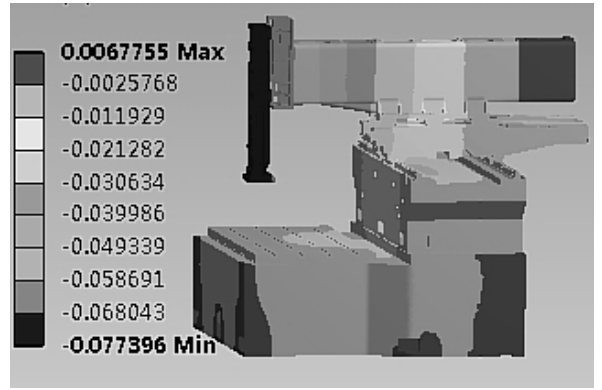

d

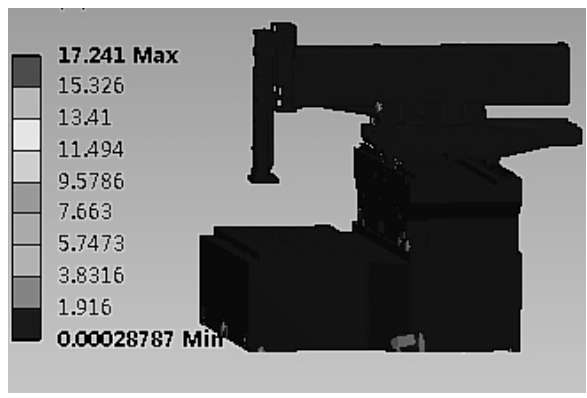

$\mathrm{e}$

Fig. 4. Deformation and stress nephograms: (a) total deformation of the whole machine; (b) $X$-direction deformation; (c) $Y$-direction deformation; (d) $Z$-direction deformation; (e) equivalent stress of whole machine.

The static analysis of the finite element model is carried out. The deformation nephograms and the equivalent stress nephogram are shown in Fig. 4. 
As is shown in the deformation nephograms (Fig. 4a-d), the majority of parts is in the dark region of smaller deformation, while the maximum deformation appears in the front half-portion of the $Z$-axis, being about 0.0837 . The deformation in the $X$ direction is uniform, its maximum value is 0.0024 , and it appears mainly at the left and right ends of the front rail. The maximum deformation in the $Y$ direction occurs at the top end of the $Z$-axis and the $Z$-axis base, its value is 0.0405 . The maximum displacement in the $Z$ direction is 0.0774 , appearing along the whole $Z$-axis. Based on the above analysis, the deformations of the worktable, bed, column, and sliding plate are relatively smaller, while the ram, $Z$-axis, and the $Z$-axis base are the mainly deformated parts. The $Z$-axis base bolted in the front of the ram can be taken as a cantilever beam. According to the characteristics of cantilever beam deformation, the $Z$-axis base deformation is mainly caused by the ram, so the $Z$-axis and ram are the main deformable components of the machine, and their stiffness in the $Y$ and $Z$ directions should be improved. This finding is exactly consistent with the actual situation due to the structure of the machine itself and deformation under loading.

Figure $4 \mathrm{e}$ shows that most of the machine equivalent stress values are below 1.916 $\mathrm{MPa}$, and the maximum value is $17.241 \mathrm{MPa}$, which is found in front of the sliding plate. The casting materials of the machine tool are made of gray cast iron, and the ultimate strength is about $250 \mathrm{MPa}$. The data show that static strength conditions meet the requirements entirely, and the machine assesses an adequate safety factor. From stress analysis, the ability of the whole machine to resist fracture is high, thus the machine design is rational.

\section{Dynamic Analysis of the Whole Machine.}

3.1. Modal Analysis. The analysis of static characteristics indicates that the stiffness in the $Y$ and $Z$ directions is inadequate, and deformation occurs mainly in the ram and $Z$-axis. For further confirmation of the weak parts, the results obtained were employed for the modal analysis of the whole machine. The natural frequency and vibration modes of the whole machine can be obtained to determine the vibration characteristics of the machine.

The process of modal analysis is similar to static analysis, so the finite element model of static analysis can be used as the model of modal analysis. Their preprocessing is much the same. The difference is that the modal analysis does not require to impose loads. Due to low-order natural vibration modes determining the dynamic characteristics, the first ten-order modes of the whole machine are chosen. The first four vibration modes are shown in Fig. 5, and the first ten-order natural frequency and vibration modes are listed in Table 2.

As can be seen from vibration modes, vibration-induced deformations of the bed and worktable are smaller than others, so their influence on the dynamic performance of the machine is not high. The vibration of the ram and $Z$-axis is evident, which will directly affect the machining precision. Among them, the swing of the local machine mainly appears in the first and second order models. From the third order, the swing and local twist occur in the $Z$-axis, ram, and other key parts. The results indicate that the $Z$-axis and ram are the main weak sides of the EDM machine, which is consistent with the static analysis.

3.2. Harmonic Response Analysis. The purpose of harmonic response analysis is to elucidate the response under the excitation force and gain the response curves of deformation versus the vibration frequency. From these curves, the corresponding peak is obtained as well as its deformation. As a result of modal analysis, the vibration modes of the system can be obtained, while analyzing harmonic response is to observe the machine vibration performance interfered with dynamic cutting force more clearly.

In this paper, the modal iteration method is used to perform harmonic response analysis. In the actual machining, the tool electrode fixed on the electrode mounting plate moves up and down to realize electric discharge machining between the electrode and workpiece. An excitation force of $1000 \mathrm{~N}$ is applied to the node, which is chosen from the center of the electrode mounting plate along the positive direction of the $Z$-axis. The fixed 
$\mathrm{T}$ a b 1 e 2

The First-Ten Order Natural Frequency and Vibration Modes of the Whole Machine

\begin{tabular}{|c|c|l||}
\hline \hline Order & $\begin{array}{c}\text { Frequency } \\
(\mathrm{Hz})\end{array}$ & \multicolumn{1}{|c||}{ Vibration mode } \\
\hline 1 & 49.300 & $\begin{array}{l}\text { Swing to the left and right of the whole upper part above the sliding plate } \\
\text { along the } X \text { direction }\end{array}$ \\
\hline 2 & 59.770 & $\begin{array}{l}\text { Swing up and down of the whole upper part above the column along the } Z \\
\text { direction }\end{array}$ \\
\hline 3 & 64.803 & Reverse twist of the $Z$-axis and ram \\
\hline 4 & 75.451 & Swing forward and backward of the $Z$-axis along the $Y$ direction \\
\hline 5 & 95.687 & Twist of the $Z$-axis and ram in the same direction \\
\hline 6 & 99.725 & Local bending of the $Z$-axis along the $Y$ direction \\
\hline 7 & 140.510 & $\begin{array}{l}\text { Reverse swing of the } Z \text {-axis, ram and column, accompanied by the local } \\
\text { twist of the } Z \text {-axis }\end{array}$ \\
\hline 8 & 143.890 & $\begin{array}{l}\text { Swing of the } Z \text {-axis, ram and column in the same direction, accompanied by } \\
\text { the local twist of the ram }\end{array}$ \\
\hline 10 & 173.460 & $\begin{array}{l}\text { Local twist of the ram } \\
\text { Local bending of the sliding plate along the } Z \text { direction }\end{array}$ \\
\hline \hline
\end{tabular}

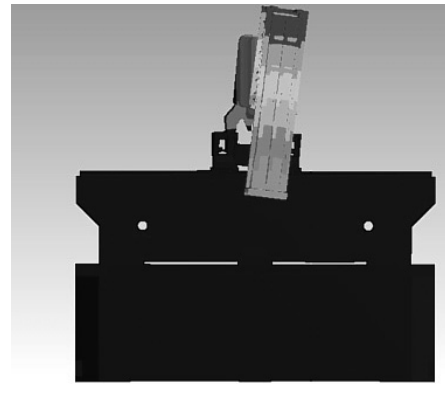

a

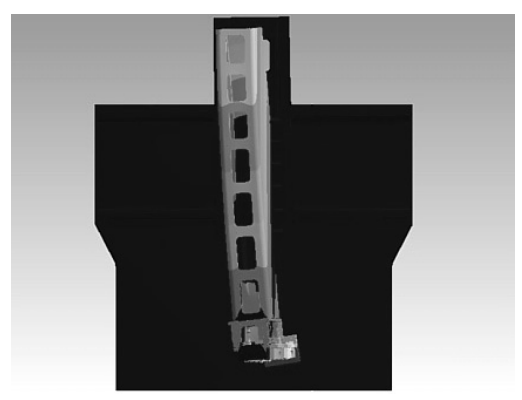

C

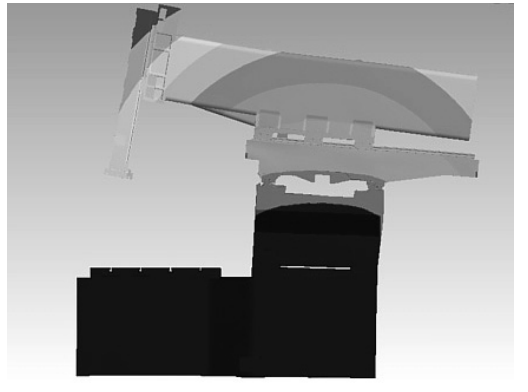

b

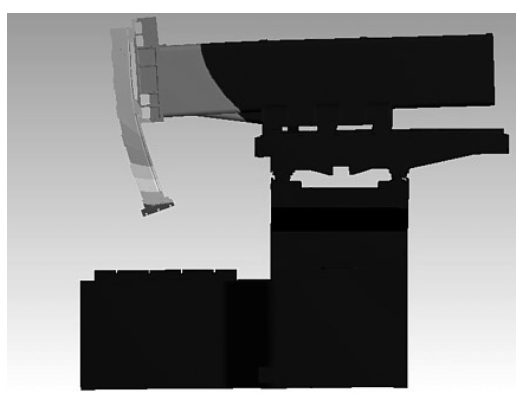

d

Fig. 5. First four-order modes of the whole machine system: (a) first-order mode; (b) second order-mode; (c) third-order mode; (d) fourth-order mode.

support is also chosen between the bottom of the bed and ground in the same way as with the previous set. According to the natural frequency obtained in modal analysis, the frequency range is set from 0 to $200 \mathrm{~Hz}$, and the "solution interval" is divided into 20 steps to achieve the harmonic response. The frequency response deformation curve is shown in Fig. 6. 


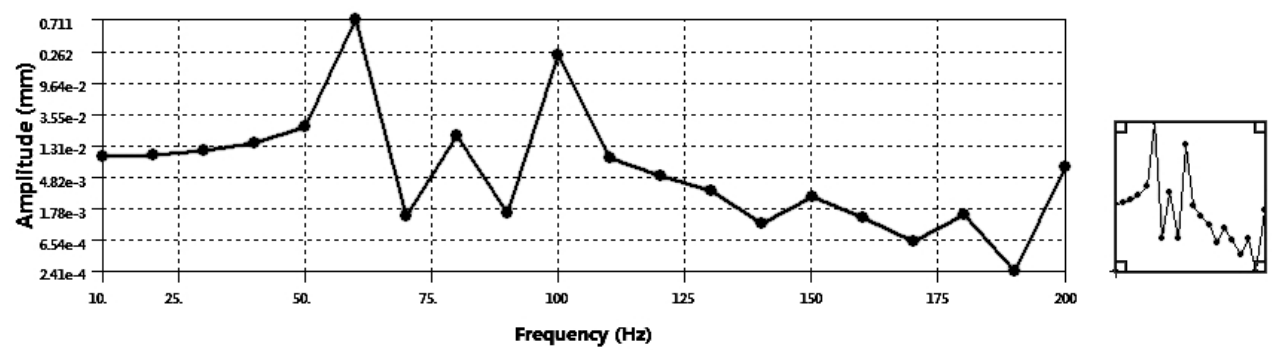

Fig. 6. Z-direction frequency response deformation curve of the selected point.

According to Fig. 6, the peak of the frequency response deformation curve appears at $60 \mathrm{~Hz}$, while the frequency value for larger deformation is $100 \mathrm{~Hz}$, which happens with the second- and sixth-order models of the system, respectively. It indicates that both models are most likely to be excited under the dynamic excitation force; thus, they have the largest impact on the dynamic performance of the EDM machine. The results gained by harmonic response analysis agree well with the vibration modes obtained by modal analysis. It shows that the upper part deformation of the machine is evident, while the stiffness of the ram and $Z$-axis in the $Y$ and $Z$ directions is small.

Conclusions. The whole process of analysis proceeds on the platform of SolidWorks and ANSYS workbench. The static and dynamic characteristics for a particular type of the EDM machine are analyzed using the finite element method. The results indicate that the $Y$ and $Z$-direction deformations of the ram and $Z$-axis are large, which means that the $Y$ - and $Z$-direction stiffness of the ram and $Z$-axis is the weak point of the whole machine. The strengthening of stiffness is the key to improve the machining precision of the EDM machine. Analysis as the element of research is an effective method to find out the weak points of the whole machine. It provides a theoretical basis and valuable reference for the structural optimization of the machine.

The future work is to pay more attention to study of topology optimization methods, and apply multidisciplinary optimization techniques to make the structure of the EDM machine more effective.

Acknowledgments. This work is supported by Innovation Project II-3: Research on the Integration of Large Depth-Diameter Ratio Micro-Hole Ultra-Precision EDM Equipment of Aero-Engine (Project No. PXM2014_178102_000006) and Jing-Hua Talents Project of Beijing University of Technology.

1. D. Y. Yang and J. J. Fu, "Development of electrical discharge forming technology," Aeronaut. Manuf. Technol., 5, 43-46 (2010).

2. A. Myers, D. G. Ford, and Q. Xu, "Finite element analysis of the structural dynamics of a vertical milling machine," in: D. G. Ford (Ed.), Laser Metrology and Machine Performance VI, WIT Press, Southampton (2003), pp. 431-440.

3. X. Zhang, D. P. Liu, and Z. Tao, "Dynamic characteristic analysis of key parts of CX8075 machining center based on ANSYS workbench," Mach. Des. Manuf., 7, 146-148 (2011).

4. F. Kang and J. W. Fan, "Dynamic characteristic analysis of CNC machine tool based on ANSYS," Mach. Des. Manuf., 7, 181-182 (2008).

5. C. G. Feng, J. J. Cao, and L. Zhang, "Research on dynamic character of milling machining centre worktable based on ANSYS workbench," Mach. Tool Hydraul., 41, No. 7, 175-177 (2013). 
6. Y. H. Cai, Y. Li, J. Y. Liu, and S. F. Wang, "Modal analysis for EDM machine tool," Electromach. Mould, 6, 74-76 (2013).

7. L. Li, The Dynamic Analysis and Optimization of a Five-Axis SEDM's Moving Link, Master Thesis, Soochow University, Jiangsu, China (2011).

8. K. K. Shi, Z. K. Xie, P. Lu, et al., "The static and dynamic characteristics analysis of HTM40100 based on ANSYS," Modul. Mach. Tool Autom. Manuf. Tech., 7, 44-45 (2013).

Received 20. 10. 2014 